\title{
Production and trapping of carbon clusters for absolute mass measurements at ISOLTRAP
}

C. Scheidenberger ${ }^{\mathrm{a}, \mathrm{b}}$, G. Bollen ${ }^{\mathrm{c}, \mathrm{a}}, \mathrm{F}$. Herfurth ${ }^{\mathrm{b}}$, A. Kellerbauer ${ }^{\text {a }}$, H.-J. Kluge ${ }^{b}$, M. Koizumi ${ }^{\text {a }}$, S. Schwarz ${ }^{\text {a }}$, L. Schweikhard ${ }^{\mathrm{d}}$

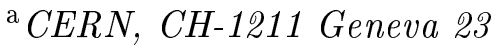

${ }^{\mathrm{b}}$ GSI, D-64291 Darmstadt

${ }^{\mathrm{c}}$ Ludwig-Maximilians Universität, D-85748 München

${ }^{\mathrm{d} J o h a n n e s ~ G u t e n b e r g-U n i v e r s i t a ̈ t, ~ D-55099 ~ M a i n z ~}$

\begin{abstract}
Singly-charged carbon clusters $\mathrm{C}_{n}^{+}(n \geq 1)$ have been produced by laser-induced desorption and fragmentation of $\mathrm{C}_{60}$ fullerenes and have been injected into and stored in the Penning trap system of the ISOLTRAP mass spectrometer at ISOLDE/CERN. The present study is the first step to extend the until now direct mass measurements at ISOLTRAP to absolute mass measurements by using clusters of ${ }^{12} \mathrm{C}$.
\end{abstract}

Key words: Fullerenes; Carbon clusters; Penning trap; Exotic nuclei; Atomic mass standard; Absolute mass measurements

\section{Introduction and motivation}

The discovery of the remarkable properties of the Buckminsterfullerene $\mathrm{C}_{60}(1)$ has stimulated a great deal of fundamental research and technological development. The ability to produce $\mathrm{C}_{60}$ in macroscopic amounts (2) and the mechanism of laser-induced desorption and fragmentation allows one to produce by very simple means free carbon clusters $\mathrm{C}_{n}, n \geq 1$, and to investigate their chemical and physical properties or to use them for applications.

One application is to use carbon clusters as mass references in high-accuracy direct mass measurements (3) at ISOLTRAP (4) at ISOLDE (5) at CERN, because $1 / 12$ of the mass of the ${ }^{12} \mathrm{C}$ atom is defined as the atomic mass unit. So far the (unknown) mass $m\left({ }^{A} X^{+}\right)$of an ion $X$ with mass number $A$ is obtained from the comparison of its cyclotron frequency $\omega_{c}$ with that of a well known "reference mass" $m($ ref $)$ through the equation $m\left({ }^{A} X^{+}\right)=$ $m($ ref $) \cdot \omega_{c}($ ref $) / \omega_{c}\left({ }^{A} X^{+}\right)$. A relative mass accuracy $\delta m / m \simeq 10^{-7}$ is routinely reached (6). It is obvious that the use of the ${ }^{12} \mathrm{C}$ mass as reference eliminates the uncertainty of the mass of the reference ion by definition. Furthermore, possible mass dependent systematic errors can be revealed and investigated for the first time. Thus the production and trapping of carbon cluster ions is the key to still higher accuracy and in particular absolute atomic mass measurements.

Preprint submitted to Elsevier Preprint $\quad 8$ May 2001 


\section{Ion source}

A schematic of the cluster ion source is shown in fig. 1. A cylindrically shaped piece of pressed $\mathrm{C}_{60}$ material (diameter $10 \mathrm{~mm}$, height $3 \mathrm{~mm}$, purity: $\left.\mathrm{C}_{60}>99.4 \%(7)\right)$ is placed at the bottom of the source on an accelerating potential of $2.70 \mathrm{kV}$ (this is the typical transport energy to the cooler trap, the first Penning trap of ISOLTRAP (4)). The generated ions are extracted with the extraction and einzel lens system at the upper end of the source. The frequency-doubled $532 \mathrm{~nm}$ beam of a Q-switched Nd:YAG laser (Lumonics HY200, maximum pulse energy $75 \mathrm{~mJ}$ at a repetition rate of $10 \mathrm{~Hz}$, pulse duration $8 \mathrm{~ns}$ ), is focused on the $\mathrm{C}_{60^{-}}$ target by a quartz lens $(\mathrm{f}=250 \mathrm{~mm})$ to give a focal spot area of approximately $1 \mathrm{~mm}^{2}$.

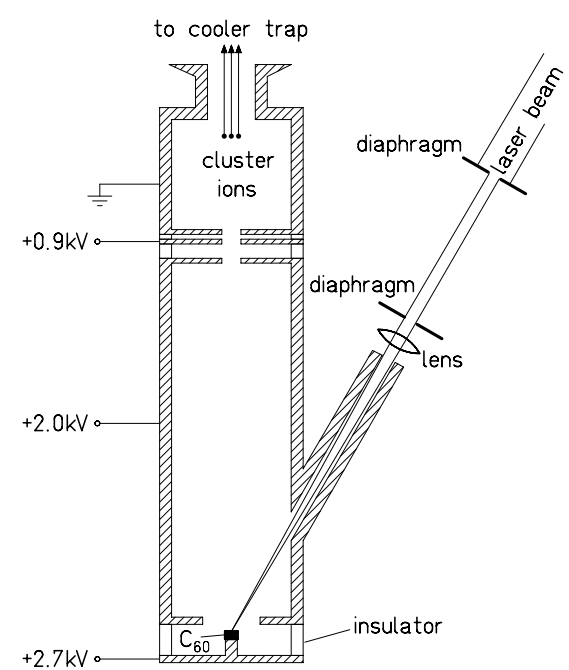

Fig. 1. Ion source used for laserinduced desorption of $\mathrm{C}_{60}$ and production of carbon clusters. The indicated voltages are optimized for extraction of $2.7 \mathrm{keV} \mathrm{C}_{5}^{+}$ions.

For the present measurements, the residual gas pressure in the source was slightly better than $1 \cdot 10^{-6}$ mbar. The source was vertically mounted under the cooler Penning trap of the ISOLTRAP setup. Positive and negative clusters were detected with the micro-channel plate detector (MCP) mounted $2.01 \mathrm{~m}$ downstream from the exit of the source directly under the cooler Penning trap. The laser is triggered at the beginning of each ISOLTRAP measuring cycle (4) and the TDC for the time-of-flight determination (TOF) is started with the same signal.

Desorption of $\mathrm{C}_{60}^{+}$sets in at a fluence of about $5 \mathrm{~mJ} / \mathrm{cm}^{2}$ and the intensity increases strongly with fluence. At higher fluences small amounts of fragment ions $\mathrm{C}_{58}^{+}, \mathrm{C}_{56}^{+}, \ldots$ are produced, mainly by successive loss of $\mathrm{C}_{2}$. The spectrum shown in fig. 2 was obtained at a fluence of about $10 \mathrm{~mJ} / \mathrm{cm}^{2}$ and shows all even- $n$ fragments down to $n=32$. The light fragments ranging from the monomer $\mathrm{C}$ up to $\mathrm{C}_{19}$ are produced either by successive emission of predominantly $\mathrm{C}_{3}$ or by direct break-up of excited $\mathrm{C}_{60}$ (8). Coalescence products appear in the mass spectrum around $\mathrm{C}_{120}^{+}$. At a fluence of about $15 \mathrm{~mJ} / \mathrm{cm}^{2}$ clusters up to $n=23$ were observed. Thus the whole mass spectrum of the chart of nuclei can be covered from mass number $A=12$ up to $A=276$ and it is always possible to choose a reference ion with a mass number close to that of the ion under investigation (when target material is used, which is made out of isotopically enriched ${ }^{13} \mathrm{C}$ (whose mass is known with extreme accuracy), one can reach even more and closer mass numbers; in particular, for all investigated ions with mass numbers $A \geq 132$ one finds a reference carbon cluster ion with the same mass number). 

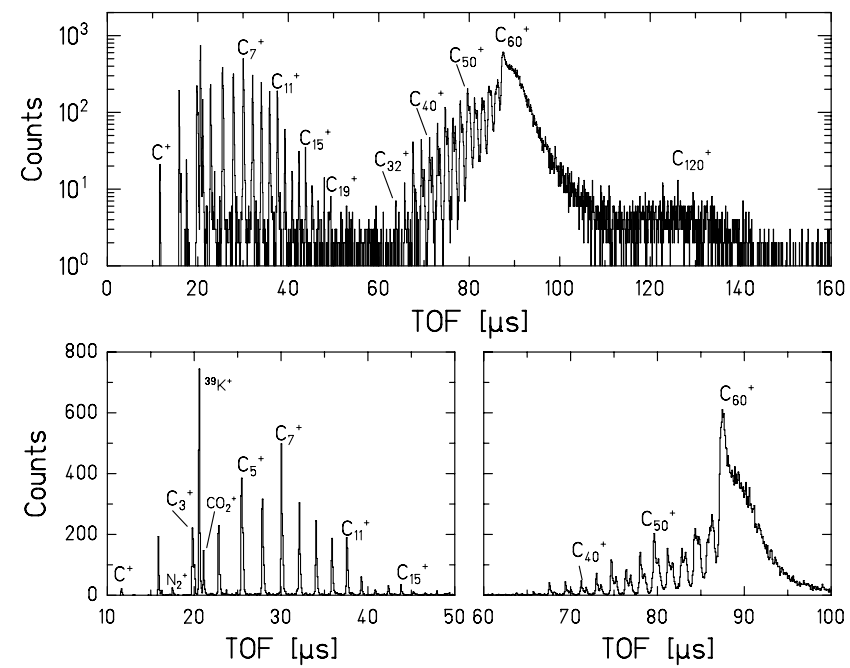

Fig. 2. Time-of-flight mass spectra of carbon cluster ions and some charged contaminants produced by laser induced desorption and fragmentation of fullerenes at $532 \mathrm{~nm}$ with a fluence of about $10 \mathrm{~mJ} / \mathrm{cm}^{2}$.

\section{Storage of clusters in the cooler Penning trap}

Carbon clusters were injected into the cooler trap and stored. With pulsed ion bunch dynamic trapping (9), $\mathrm{C}_{5}^{+}$ions were selected for capture into the cooler Penning trap. Helium buffer gas cooling without rf-excitation was applied (9) and the storage time in the trap was varied between $5 \mathrm{~ms}$ and $390 \mathrm{~ms}$. After ejection out of the cooler Penning trap the ions were detected with a MCP detector placed above the cooler trap. A time-of-flight spectrum of ions ejected after $370 \mathrm{~ms}$ storage time is shown in the left part of fig. $3 . \mathrm{C}_{5}^{+}$ions form the most prominent peak. Smaller clusters $\mathrm{C}_{n=2,3,4}$ are produced either due to fragmentation from heavier hot clusters or due to dissociation by buffer-gas collisions. Single carbon ions are not observed. The origin of heavier clusters $\mathrm{C}_{n=6,7}$ is presently not clear. One reason might be an imperfect mass selection in the injection and capture process.

After $35 \mathrm{~ms}$ storage time the width of all peaks in the TOF spectrum reaches a constant minimum value indicating that thermal equilibrium of the cooling process is reached. In the right part of fig. 3 the intensity distribution of the
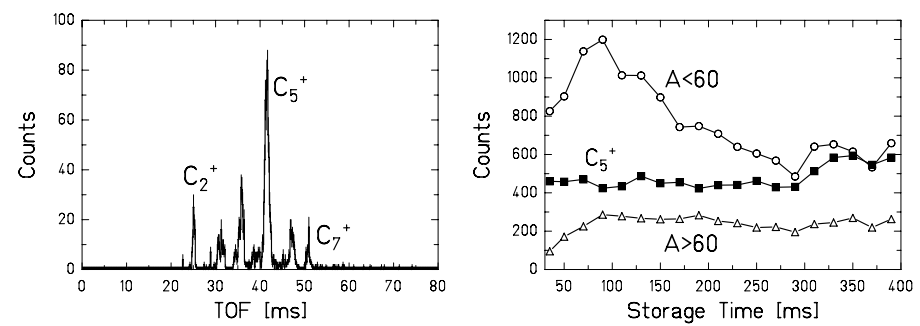

Fig. 3. Left: time-of-flight spectrum of carbon clusters ejected after $370 \mathrm{~ms}$ storage time (see text). Right: number of ejected ions with mass numbers $A<60$ (circles), $A>60$ (triangles), and $\mathrm{C}_{5}^{+}$-ions (full squares) as a function of storage time. Within the shown time span of $355 \mathrm{~ms}$ no losses of $\mathrm{C}_{5}^{+}$-ions are observed.

clusters is shown as a function of storage time. Clusters lighter $(A<60)$ and heavier $(A>60)$ than $\mathrm{C}_{5}^{+}(A=60)$ have been grouped for simplicity. Their 
intensity distributions exhibit a time dependence which cannot be interpreted easily. This will be an issue of forthcoming and more detailed investigations. However, as an important result one notes that the number of $\mathrm{C}_{5}^{+}$ions is almost independent of storage time, i. e., after cooling no losses are observed within a storage time as long as $355 \mathrm{~ms}$.

\section{Summary and outlook}

Carbon cluster ions $\mathrm{C}_{n}^{+}(n \geq 1)$ have been produced by laser-induced desorption. $\mathrm{C}_{5}^{+}$ions were trapped and buffer-gas cooled in the cooler Penning trap of the ISOLTRAP setup for periods longer than $350 \mathrm{~ms}$ without losses. This is the first step on the way to use carbon cluster ions as reference ions for high-accuracy mass measurements, which has several advantages:

1) Absolute mass measurements can be performed instead of direct ones.

2) The upper limit of the mass dependent error has been estimated for the ISOLTRAP spectrometer to be $\delta m / m \simeq 2 \cdot 10^{-9}$ per mass unit difference between the investigated and the reference ion (6). With carbon cluster ions it is always possible to choose a reference mass which is at most six mass units away from the investigated ion. Therefore the mass dependent error will always satisfy the relation $\delta m / m \leq 1.2 \cdot 10^{-8}$.

3) The origin of this error is expected to be mainly due to spatial imperfections of the electric and magnetic field inside the precision trap. With cluster ions it will be possible for the first time to reveal and to investigate this error systematically over a wide mass range.

4) With this type of ion source one can easily provide carbon clusters which are accelerated to kinetic energies of the order of several $10 \mathrm{keV}$, a feature which can be useful for in-flight mass spectrometers such as for instance MISTRAL (10).

\section{Acknowledgement}

Special thanks to H. Riege, who provided the ion-source chamber. Fruitful and stimulating discussions with E. E. B. Campbell are gratefully acknowledged. One of us (C. S.) would like to thank CERN for a one-year Scientific Associateship and the ISOLDE collaboration for the kind hospitality during the stay.

\section{References}

[1] H. W. Kroto et al., Nature 318 (1985) 162.

[2] W. Krätschmer et al., Nature 347 (1990) 354.

[3] M. Lindinger et al., Z. Phys. D 20 (1991) 441.

[4] G. Bollen et al., Nucl. Instr. Meth. A 368 (1996) 675.

[5] E. Kugler et al., Nucl. Instr. Meth. B 70 (1992) 41.

[6] D. Beck et al., Nucl. Instr. Meth. B 126 (1997) 374.

[7] Hoechst AG, D-65926 Frankfurt/Main, Germany.

[8] R. Vandenbosch, Phys. Rev. A 59 (1999) 3584.

[9] H. Raimbault-Hartmann et al., Nucl. Instr. Meth. B 126 (1997) 378.

[10] M. D. Lunney et al., Hyp. Int. 99 (1996) 105. 